\title{
Unexpected cytotoxicity of a triisopropylsilylated syringaldehyde derived cinnamic acid amide
}

\author{
Björn A. Weber ${ }^{1}$, Ratna Kancana Wolfram ${ }^{1}$, Sophie Hoenke ${ }^{1,}$ Lucie Fischer $^{1}$, Ahmed Al-Harrasi ${ }^{2}$ and \\ René Csuk 1,* \\ ${ }^{1}$ Full Address: Martin-Luther-University Halle-Wittenberg, Organic Chemistry, Kurt-Mothes-Str. 2, D-06120 \\ Halle (Saale), Germany \\ ${ }^{2}$ Full Address: University of Nizwa, Chair of Oman's Medicinal Plants and Marine Natural Products, P.O. Box \\ 33, PC 616, Birkat Al-Mauz, Nizwa, Sultanate of Oman
}

\begin{abstract}
A small series of substituted cinnamic acid amides was prepared and screened for their cytotoxic activity. As a rather astonishing and unprecedented result, compound $\mathbf{5}$ holding a triisopropylsilyl (TIPS) protecting group at position 4 of the aromatic ring was highly cytotoxic $\left(\mathrm{EC}_{50}=3.2 \mu \mathrm{M}\right.$ for HT29 human colon adenocarcinoma cells) while analogs with a methoxy or hydroxyl group at this position were of low cytotoxicity or not cytotoxic at all.
\end{abstract}

Keywords: cinnamic acid amides; cytotoxicity; SRB assay; HT29 cells.

\section{Introduction}

Cinnamic acid derivatives are widely used as flavors and fragrance ${ }^{1,2}$. During the last years, compounds holding a cinnamoyl scaffold attracted attention because of their biological activities combined with low toxicity ${ }^{3-8}$. However, several of these compounds have also shown cytotoxic ${ }^{9-14}$ as well as antimicrobial ${ }^{15-20}$ and anti-oxidant properties ${ }^{21-26}$. Also, cinnamic acid derivatives have been used as valuable starting materials for the synthesis of peroxisome proliferatoractivated receptors (PPAR). This class of compounds is of high scientific and commercial interest for the therapy of patients suffering from diabetes mellitus type II 27,28 .

\section{Results and discussion}

During our research on analogs of piplartine ${ }^{13,29}$, we became interested in the synthesis of several amides of 4-hydroxy-3,5-dimethoxy-cinnamic acid and their biological properties, especially their cytotoxic impact on human tumor cell lines.

Reaction (Scheme 1) of syringaldehyde (1, 4hydroxy-3,5-dimethoxy-benzaldehyde) with triisopropylsilyl chloride (TIPS-Cl) gave $93 \%$ of TIPS-protected $2^{30}$. Its reaction with malonic acid under Knoevenagel-Doebner conditions furnished $(E)$ configurated cinnamic acid $\mathbf{3}$. This compound is characterized in its ${ }^{1} \mathrm{H}$ NMR spectrum by the presence of two doublets at $\delta=7.61$ and $6.38 \mathrm{ppm}$ with a coupling constant ${ }^{3} \mathrm{~J}=15.9 \mathrm{~Hz}$ being typical for $(E)$ configurated olefins. The corresponding carbons were detected in the ${ }^{13} \mathrm{C}$ NMR spectrum at $\delta=147.1$ and $117.1 \mathrm{ppm}$, respectively. The reaction of piperidine with 2-chloro-ethanol gave $4^{31-34}$ whose reaction with $\mathbf{3}$ afforded $\mathbf{5}$ as a colorless solid in $92 \%$ isolated yield. Desilylation of $\mathbf{5}$ with tetra- $n$-butylammonium fluoride trihydrate in THF yielded 6. Monobocylation of piperazine (7) gave mono- $N$-bocylated $8^{35}$ whose reaction with 2-bromo-ethanol yielded 9. From the reaction of $\mathbf{9}$ with $\mathbf{3}$ compounds $\mathbf{1 0}$ was obtained. The reaction of $(E)$ 3,4,5-trimethoxycinnamic acid (11) with 4 gave compound 12 in $90 \%$ isolated yield while its reaction with $\mathbf{9}$ afforded $\mathbf{1 3}$. For comparison $(E)$ cinnamic acid $\mathbf{1 4}$ was transformed into esters $\mathbf{1 5}$ and 16, respectively.

The compounds were subjected to sulforhodamine B assays (SRB) to determine their cytotoxic activity employing several human tumor cell lines (FaDu pharynx carcinoma, A2780 ovarian carcinoma, HT29 colon adenocarcinoma, MCF-7 breast adenocarcinoma and SW1736 thyroid gland carcinoma); the results of this assays are summarized in Table 1. 
<smiles>COc1cc(C=O)cc(OC(=O)COc2c(OC)cc(C=O)cc2OC)c1O</smiles><smiles>[R5]Oc1c(OC)cc(/C=C/C(=O)OCCN2CCCCC2)cc1OC</smiles>

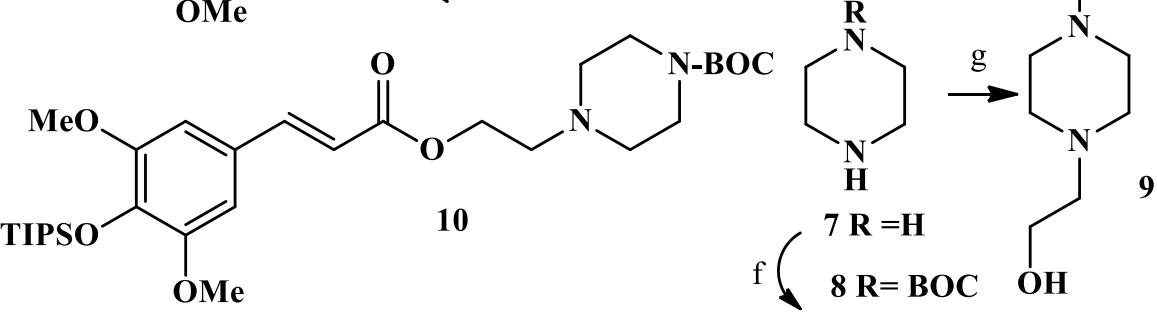<smiles>[R]O[R]([H])=[V]</smiles>

Scheme 1. Synthesis of 2-16

$$
\begin{aligned}
& 12 \mathrm{R}=\mathrm{OMe}, \mathrm{X}=\mathrm{CH} 2 \\
& 13 \mathrm{R}=\mathrm{OMe}, \mathrm{X}=\mathrm{N}-\mathrm{BOC} \\
& 15 \mathrm{R}=\mathrm{H}, \mathrm{X}=\mathrm{CH} 2 \\
& 16 \mathrm{R}=\mathrm{H}, \mathrm{X}=\mathrm{N}-\mathrm{BOC}
\end{aligned}
$$

Reactions and conditions: a) TIPS- $\mathrm{Cl}$, triethylamine, cat. DMAP, DCM, $25^{\circ} \mathrm{C}, 1 \mathrm{~h}, 93 \%$; b) malonic acid, pyridine, cat. piperidine, $120^{\circ} \mathrm{C}, 1 \mathrm{~h}, 62 \%$; c) $\mathrm{Cl}-\mathrm{CH}_{2}-$ $\mathrm{CH}_{2}-\mathrm{OH}$, piperidine, toluene, $70^{\circ} \mathrm{C}, 5 \mathrm{~h}, 92 \%$; d) EDC, 4, cat. DMAP, DCM, $25^{\circ} \mathrm{C}, 1$ day, $92 \%$; e) $n$ $\mathrm{Bu}_{4} \mathrm{NF}$ trihydrate, $\mathrm{THF}, 25^{\circ} \mathrm{C}, 6 \mathrm{~h}, 87 \%$; f) according to lit. ${ }^{35}, 71 \%$; g) $\mathrm{Br}-\mathrm{CH}_{2}-\mathrm{CH}_{2}-\mathrm{OH}$, tritehylamine,<smiles>COc1cc(/C=C/C(=O)O)cc(OC)c1O</smiles>

3<smiles>OCCN1CCC(CN2CCCCC2)CC1</smiles>

Table 1. Cytotoxicity of compounds 1-3, 5, 6, 11-16.

\begin{tabular}{|c|c|c|c|c|c|}
\hline$\#$ & FaDu & A2780 & HT29 & MCF-7 & SW1736 \\
\hline $\mathbf{1 - 3}$ & $>30$ & $>30$ & $>30$ & $>30$ & $>30$ \\
\hline $\mathbf{5}$ & $6.0 \pm 0.2$ & $5.8 \pm 0.3$ & $3.2 \pm 0.7$ & $4.4 \pm 0.4$ & $9.3 \pm 0.9$ \\
\hline $\mathbf{6}$ & $27.1 \pm 1.7$ & $28.3 \pm 2.3$ & $29.9 \pm 2.4$ & $25.1 \pm 3.2$ & $29.1 \pm 3.0$ \\
\hline $\mathbf{1 1 - 1 6}$ & $>30$ & $>30$ & $>30$ & $>30$ & $>30$ \\
\hline STS & $0.14 \pm 0.01$ & $0.12 \pm 0.01$ & $0.15 \pm 0.02$ & $0.10 \pm 0.02$ & $0.12 \pm 0.02$ \\
\hline
\end{tabular}

$\mathrm{EC}_{50}$ values from SRB assays after $96 \mathrm{~h}$ of treatment are given in $\mu \mathrm{M}$; the values are averaged from three independent experiments each performed in triplicate; confidence interval CI $=95 \%$; cut-off $30 \mu \mathrm{M}$. Human tumor cell lines: FaDu pharynx carcinoma, A2780 ovarian carcinoma, HT29 colon adenocarcinoma, MCF-7 breast adenocarcinoma and SW1736 thyroid gland carcinoma. Staurosporine (STS) was used as a positive control.

As a result, 11-16 were not cytotoxic within the limits of the assay (cut-off $30 \mu \mathrm{M}$ ). The highest (and rather
DCM, $60^{\circ} \mathrm{C}, 12 \mathrm{~h}, 70 \%$; h) EDC, 9, cat. DMAP, $\mathrm{DCM}, 25^{\circ} \mathrm{C}, 1$ day, $73 \%$; i) 12 from 11: EDC, EDC, 4, cat. DMAP, DCM, $25^{\circ} \mathrm{C}, 1$ day, $86 \%$; j) 13 from 11: EDC, EDC, 9, cat. DMAP, DCM, $25^{\circ} \mathrm{C}, 1$ day, 61\%; k) 15 from 14: EDC, EDC, 4, cat. DMAP, DCM, $25^{\circ} \mathrm{C}, 1$ day, 90\%; 16 from 14: EDC, 9, cat. DMAP, DCM, $25^{\circ} \mathrm{C}, 1$ day, $62 \%$.

unexpected) cytotoxicity was established for $\mathbf{5}$ showing $\mathrm{EC}_{50}$ values in the low $\mu \mathrm{M}$ range for the human tumor cell lines. This compound was shown to be most cytotoxic for human colon adenocarcinoma cells HT29, and an $\mathrm{EC}_{50}=3.2 \mu \mathrm{M}$ was determined. Interestingly, most of the cytotoxicity is lost for desilylated 6. The reason for this loss of cytotoxicity cannot be explained by the mere presence of a free phenolic hydroxy group in compound $\mathbf{6}$. Compounds 12 (with a methoxy group on the phenyl substituent) and $\mathbf{1 5}$ (only hydrogen substituents on the ring) have no cytotoxicity either. Thus, the reason for the 
enhanced cytotoxicity of $\mathbf{5}$ remains unclear and will be subject to further studies concerning also the modeof-action for this class of compounds.

\section{Conclusion}

Several cinnamic acid derived amides were prepared and screened for their cytotoxic activity employing five human tumor cell lines. Interestingly, a piperidinyl-ethyl substituted 3,5-dimethoxy cinnamic acid amide $\mathbf{5}$ carrying a TIPS protecting group at position 4 was highly cytotoxic for all tumor cell lines in the low $\mu \mathrm{M}$ concentration range. While analogs holding a hydroxyl group (as exemplified in 6) or a methoxy substituent (as exemplified in 12) or no substituents (as exemplified in 15) at this position were of significantly reduced cytotoxicity or were not cytotoxic at all.

\section{Acknowledgments}

Many thanks are due to Dr. D. Ströhl and his team for the NMR spectra as well as to Dr. R. Kluge for numerous ESI-MS spectra. The IR and UV/Vis spectra were recorded by Ms. V. Simon. Additional help in the lab has been provided by Ms. J. Wiese, Ms T.L.T. Luong, and especially by Ms T. Zeitz. The cell lines were kindly provided by Dr. Th. Müller (Dep. of Haematology/Oncology, Martin-Luther-Universität Halle-Wittenberg). The authors declare no conflict of interest.

\section{Experimental}

Melting points are uncorrected (Leica hot stage microscope), NMR spectra were recorded using the Varian spectrometer Gemini 2000 ( $\delta$ given in ppm, $J$ in $\mathrm{Hz}$ ), MS spectra were taken on a Finnigan MAT LCQ 7000 (electrospray, voltage $4.5 \mathrm{kV}$, sheath gas nitrogen) instrument. TLC was performed on silica gel (Merck 5554); elemental analyses were performed on a Vario EL (CHNS). The solvents were dried according to usual procedures (DCM by distillation over $\mathrm{K}_{2} \mathrm{CO}_{3}$, pyridine over $\mathrm{KOH}$, toluene over $\mathrm{Na}$ ). The purity of the compounds was determined by HPLC and found to be $>97 \%$.

\section{Sulforhodamine B assay (SRB)}

The cytotoxicity of the compounds was evaluated using the sulforhodamine-B (Kiton-Red S, ABCR) micro culture colorimetric assay. Cells were seeded into 96-well plates on day 0 at appropriate cell densities to prevent confluence of the cells during the period of the experiment. After 24 hours, the cells were treated with six different concentrations $(1,3,7$, $12,20$ and $30 \mu \mathrm{M})$ minimum. The final concentration of DMSO/DMF never exceeded $0.5 \%$, which was non-toxic to the cells. After a $96 \mathrm{~h}$ treatment, the supernatant medium from the 96-well plates was discarded, the cells were fixed with $10 \%$ trichloroacetic acid (TCA) and allowed to rest at $4{ }^{\circ} \mathrm{C}$. After $24 \mathrm{~h}$ fixation, the cells were washed in a strip washer and dyed with SRB solution $(100 \mu \mathrm{L}, 0.4 \%$ in
$1 \%$ acetic acid) for about 20 min. After dying, the plates were washed four times with $1 \%$ acetic acid to remove the excess of the dye and allowed to air-dry overnight. Tris base solution $(200 \mu \mathrm{L}, 10 \mathrm{mM})$ was added to each well and absorbance was measured at $\lambda=570 \mathrm{~nm}$ using a 96 well plate reader (Tecan Spectra, Crailsheim, Germany). The $\mathrm{EC}_{50}$ values were averaged from three independent experiments performed each in triplicate calculated from semilogarithmic dose-response curves applying a nonlinear 4P Hills-slope equation (GraphPad Prism5; variables top and bottom were set to 100 and 0 , respectively).

\section{3,5-Dimethoxy-4-[(triisopropyl)silyloxy]- benzaldehyde (2)}

To a solution of 4-hydroxy-3,5-dimethoxybenzaldehyde (1, $9.0 \mathrm{~g}, 49.4 \mathrm{mmol})$ and triethylamine (9.0 $\mathrm{mL}, 64.6 \mathrm{mmol})$ in dry DCM $(50 \mathrm{~mL})$ triisopropyl chloride (11.80 g, $0.06 \mathrm{~mol}$ ) was slowly added at $25^{\circ} \mathrm{C}$. A catytic amount of DMAP was added, and stirring at $25^{\circ} \mathrm{C}$ was continued for 1 hour. Usual aqueous workup (water, brine, $\mathrm{MgSO}_{4}$ ) followed by column chromatography (silica gel, hexane/ethyl acetate, 5:1) gave $2(15.6 \mathrm{~g}, 93 \%)$ as a slightly yellowish oil; $\mathrm{R}_{\mathrm{F}}=0.48$ (silica gel, hexane/ethyl acetate, $5: 1$ ); b.p. $340^{\circ} \mathrm{C}$ (1 bar);

IR (film): $v=3449 \mathrm{br}, 2945 \mathrm{~m}, 2868 \mathrm{~m}, 2360 \mathrm{w}, 1695 \mathrm{~m}$, $1585 m, 1507 s, 1465 m, 1425 w, 1389 w, 1338 s, 1268 w$, $1230 \mathrm{w}, 1130 \mathrm{~s} \mathrm{~cm}^{-1}$; UV/vis $\left(\mathrm{CHCl}_{3}\right): \lambda_{\max }(\log \varepsilon)=$ 232 (4.09), 311 (4.09) nm;

${ }^{1} \mathrm{H}$ NMR (500 MHz, CDCl3): $\mathrm{d}=9.81(s, 1 \mathrm{H}, 1-\mathrm{H}$, $\mathrm{CHO}), 7.08$ ( $s, 2 \mathrm{H}$, arom.), $3.85\left(s, 6 \mathrm{H}, 2 \times \mathrm{OCH}_{3}\right)$, $1.25(m, 3 \mathrm{H}, 3 \times \mathrm{CH}-\mathrm{Si}), 1.07(d, J=7.4 \mathrm{~Hz}, 18 \mathrm{H}, 6 \times$ $\left.\mathrm{CH}_{3}\right) \mathrm{ppm}$;

${ }^{13} \mathrm{C}$ NMR (125 MHz, $\left.\mathrm{CDCl}_{3}\right): \delta=191.5(\mathrm{CH}, \mathrm{C}=\mathrm{O})$, $151.8\left(\mathrm{C}, 2 \mathrm{x}\right.$ arom. $\left.C-\mathrm{OCH}_{3}\right), 141.3(\mathrm{C}$, arom. C-OSi), 128.8 (C, arom.), 106.6 ( 2 x CH, arom. $), 55.6$ $\left(2 \times \mathrm{OCH}_{3}\right), 17.8\left(6 \times \mathrm{CH}_{3}\right), 13.9(\mathrm{CH}, 3 \times \mathrm{CH}-\mathrm{Si})$ ppm;

MS (ESI, $\mathrm{MeOH}): \mathrm{m} / \mathrm{z}(\%)=339.1\left([\mathrm{M}+\mathrm{H}]^{+}, 100\right)$; analysis calcd for $\mathrm{C}_{18} \mathrm{H}_{30} \mathrm{O}_{4} \mathrm{Si}$ (338.52): C 63.87, H 8.93; found: C 63.55, H 9.15.

\section{(E) 3-[(3,5-dimethoxy-4-[(triisopropylsilyl)oxy]- phenyl]-acrylic acid (3)}

Reaction of malonic acid $(3.2 \mathrm{~g}, 30.75 \mathrm{mmol})$ with 2 $(8.0 \mathrm{~g}, 23.6 \mathrm{mmol})$ in dry pyridine $(100 \mathrm{~mL})$ in the presence of a cat. amount of piperidine at $120^{\circ} \mathrm{C}$ for 1 hour followed by usual work-up $(10 \%$ aq. $\mathrm{HCl}$, extraction with DCM) and column chromatography (silica gel, hexane/ethyl acetate/acetic acid, 75:25:1) gave 3 (5.58 g, 62\%) as a colorless solid; m.p. 172$176^{\circ} \mathrm{C} ; \mathrm{R}_{\mathrm{F}}=0.36$ (silica gel, hexane/ethyl acetate, $4: 1)$;

IR $(\mathrm{KBr}): v=3447 \mathrm{br}, 2943 m, 2862 m, 1685 m, 1630 m$, $1582 m, 1508 s, 1458 m, 1287 s, 1133 s, 908 m, 883 m$, $671 \mathrm{~m} \mathrm{~cm}^{-1} ; \mathrm{UV} / \mathrm{vis}(\mathrm{MeOH}): \lambda_{\max }(\log \varepsilon)=336(4.16)$, $322(4.19) \mathrm{nm}$;

${ }^{1} \mathrm{H}$ NMR $\left(400 \mathrm{MHz}, \mathrm{CDCl}_{3}\right): \delta=7.61(d, J=15.9 \mathrm{~Hz}$, $1 \mathrm{H}, 3-\mathrm{H}), 6.88$ (s, 2H, arom.), $6.38(d, J=15.9 \mathrm{~Hz}$, 
$1 \mathrm{H}, 2-\mathrm{H}), 3.83\left(s, 6 \mathrm{H}, 2 \times \mathrm{OCH}_{3}\right), 1.26(m, 3 \mathrm{H}, 3 \times$ $\mathrm{CH}-\mathrm{Si}), 1.09\left(d, J=7.4 \mathrm{~Hz}, 18 \mathrm{H}, 3 \times \mathrm{CH}_{3}\right) \mathrm{ppm}$;

${ }^{13} \mathrm{C}$ NMR (125 MHz, CD $\left.{ }_{3} \mathrm{OD}\right): \delta=170.8(\mathrm{C}-1, \mathrm{C}=\mathrm{O})$, $153.1\left(\mathrm{C}, 2 \mathrm{x}\right.$ arom. $\left.C-\mathrm{OCH}_{3}\right), 147.1(\mathrm{CH}, \mathrm{C}-3), 138.5$ (C, arom. $C$-OSi), $128.6(\mathrm{C}$, arom.), $117.1(\mathrm{CH}, \mathrm{C}-2)$, $106.5(\mathrm{CH}$, arom. $), 56.2\left(2 \times \mathrm{OCH}_{3}\right), 18.7\left(6 \times \mathrm{CH}_{3}\right)$, 15.0 ( 3 x CH-Si) ppm;

MS (ESI, MeOH): m/z $(\%)=381.1\left([\mathrm{M}+\mathrm{H}]^{+}, 100\right)$; analysis calcd for $\mathrm{C}_{20} \mathrm{H}_{32} \mathrm{O}_{5} \mathrm{Si}$ (380.55): C 63.12, H 8.48; found: 62.95, H 8.69.

\section{2-(Piperidin-1-yl)-ethan-1-ol (4)}

To a solution of 2-chloro-ethanol $(8.0 \mathrm{~g}, 99.4 \mathrm{mmol})$ in dry toluene $(20 \mathrm{~mL})$, piperidine $(17.0 \mathrm{~g}, 199.6$ $\mathrm{mmol}$ ) was slowly added. The mixture was stirred at $70{ }^{\circ} \mathrm{C}$ for an additional 5 hours. The precipitate was filtered off, the volatiles were removed under reduced pressure, and the remaining oil was distilled under reduced pressure to afford $4(11.8 \mathrm{~g}, 92 \%)$ as a colorless oil; b.p. $72-76^{\circ} \mathrm{C}$ (11 mbar) [lit.: ${ }^{31}$ 204$208^{\circ} \mathrm{C}$ (706 Torr)]; $\mathrm{R}_{\mathrm{F}}=0.35$ (silica gel, $\mathrm{DCM} / \mathrm{MeOH} /$ triethylamine, 98:2:1);

IR (film): $v=3384 b r, 2935 s, 2855 m, 2803 m, 21659 w$, $1443 m, 1303 m, 1155 m, 1123 m, 1049 m, 757 \mathrm{~m} \mathrm{~cm}^{-1}$; ${ }^{1} \mathrm{H}$ NMR $\left(400 \mathrm{MHz}, \mathrm{CDCl}_{3}\right): \delta=4.02(\mathrm{OH}), 3.57(\mathrm{~m}$, $\left.2 \mathrm{H}, \mathrm{CH}_{2} \mathrm{OH}\right), 2.46\left(m, 2 \mathrm{H}, \mathrm{N}-\mathrm{CH}_{2}\right), 2.41(m, 4 \mathrm{H}$, $\left.\mathrm{N}-\mathrm{CH}_{2}\right), 1.16\left(m, 4 \mathrm{H}, \mathrm{CH}_{2}\right), 1.43\left(m, 2 \mathrm{H}, \mathrm{CH}_{2}\right) \mathrm{ppm}$; ${ }^{13} \mathrm{C} \mathrm{NMR}\left(100 \mathrm{MHz}, \mathrm{CDCl}_{3}\right): \square=59.9\left(\mathrm{~N}_{-}-\mathrm{CH}_{2}\right), 57.7$ $\left(\mathrm{O}-\mathrm{CH}_{2}\right), 54.3\left(\mathrm{~N}-\mathrm{CH}_{2}\right), 26.0\left(\mathrm{CH}_{2}\right), 24.3\left(\mathrm{CH}_{2}\right) \mathrm{ppm}$; ESI-MS (MeOH): m/z (\%)) 130.1 ([M+H $\left.]^{+}, 100\right)$; analysis calcd for $\mathrm{C}_{7} \mathrm{H}_{15} \mathrm{NO}$ (129.20): C 65.07, H 11.70, N 10.84; found: C 64.85, H 11.95, N 10.63.

(E) 2-(Piperidin-1-yl)-ethyl 3-[3,5-dimethoxy-4[(triisopropylsilyl)oxy]phenyl]-acrylate (5)

Following the procedure given for the preparation of 15, from 3 (2.69 g, $7.07 \mathrm{mmol})$ and 4 (1.29 g, 9.98 mmol), 5 (3.2 g, 92\%) was obtained as a colorless solid; m.p. $64-66^{\circ} \mathrm{C} ; \quad \mathrm{R}_{\mathrm{F}}=0.34$ (silica gel, hexane/ethyl acetate/triethylamine, 100:20:2.5);

IR (KBr): $v=3448 b r, 2940 m, 2864 m, 1697 s, 1578 m$, $1507 s, 1466 m, 1422 m, 1264 s, 1128 s, 1085 m, 900 m$, $459 \mathrm{~m} \mathrm{~cm}^{-1} ; \mathrm{UV} / \mathrm{vis}(\mathrm{MeOH}): \lambda_{\max }(\log \varepsilon)=248$ (4.10), 360 (4.17) nm;

${ }^{1} \mathrm{H}$ NMR $\left(400 \mathrm{MHz}, \mathrm{CDCl}_{3}\right): \delta=7.58(d, J=15.9 \mathrm{~Hz}$, $1 \mathrm{H},=\mathrm{CH}), 6.71(s, 2 \mathrm{H}$, arom. $), 6.32(d, J=15.9 \mathrm{~Hz}$, $1 \mathrm{H},=\mathrm{CH}), 4.32\left(t, J=6.1 \mathrm{~Hz}, 2 \mathrm{H}, \mathrm{O}-\mathrm{CH}_{2}\right), 3.81(s$, $6 \mathrm{H}, \mathrm{OMe}), 2.67\left(t, J=6.1 \mathrm{~Hz}, 2 \mathrm{H}, \mathrm{N}-\mathrm{CH}_{2}\right), 2.47(t, J$ $\left.=5.4 \mathrm{~Hz}, 4 \mathrm{H}, \mathrm{N}-\mathrm{CH}_{2}\right), 1.60\left(m, 4 \mathrm{H}, \mathrm{CH}_{2}\right), 1.44(m$, $\left.2 \mathrm{H}, \mathrm{CH}_{2}\right), 1.25$ (sept $\left., J=15.6 \mathrm{~Hz}, 3 \mathrm{H}, \mathrm{CH}\right), 1.06(d, J$ $=15.6 \mathrm{~Hz}, 18 \mathrm{H}, \mathrm{Me}) \mathrm{ppm}$;

${ }^{13} \mathrm{C}$ NMR $\left(100 \mathrm{MHz}, \mathrm{CDCl}_{3}\right): \delta=167.1\left(\mathrm{CO}_{2} \mathrm{R}\right)$, 151.4 (arom.), 145.3 (=CH), 137.5, 126.6 (arom.), 115.7 (=CH), 105.1 (arom.), $61.9\left(\mathrm{~N}-\mathrm{CH}_{2}\right), 57.5(\mathrm{O}-$ $\left.\mathrm{CH}_{2}\right), 55.5(\mathrm{OMe}), 54.8\left(\mathrm{~N}-\mathrm{CH}_{2}\right), 25.9\left(\mathrm{CH}_{2}\right), 24.2$ $\left(\mathrm{CH}_{2}\right), 17.8(\mathrm{Me}), 13.3(\mathrm{CH}) \mathrm{ppm}$;

ESI-MS $(\mathrm{MeOH}): \mathrm{m} / \mathrm{z}(\%)=492.3([\mathrm{M}+\mathrm{H} \mid+, 100)$; analysis calcd for $\mathrm{C}_{27} \mathrm{H}_{45} \mathrm{SiNO}_{5}$ (491.74): C 66.95, H 9.22, N 2.85; found: C 66.72, H 9.49, N 2.69.
(E) 2-(Piperidin-1-yl) 3-(4-hydroxy-3,5-dimethoxyphenyl) acrylate (6)

To a solution of $5(250 \mathrm{mg}, 0.51 \mathrm{mmol})$ in THF (10 $\mathrm{mL}) n-\mathrm{Bu}_{4} \mathrm{NF} * 3 \mathrm{H}_{2} \mathrm{O}(160 \mathrm{mg}, 0.51 \mathrm{mmol})$ was added, and the mixture was stirred at $25^{\circ} \mathrm{C}$ for 6 hours. Usual aqueous workup (water, brine, $\mathrm{MgSO}_{4}$ ) followed by column chromatography (silica gel, $\left.\mathrm{CHCl}_{3} / \mathrm{MeOH}, 97: 3\right)$ gave $6(148 \mathrm{mg}, 87 \%)$ as a colorless oil; $\mathrm{R}_{\mathrm{F}}=0.17$ (silica gel, $\mathrm{CHCl}_{3} / \mathrm{MeOH}$, 97:3);

${ }^{1} \mathrm{H}$ NMR (400 MHz, $\left.\mathrm{CDCl}_{3}\right): \delta=8.05(b r, 1 \mathrm{H}, \mathrm{OH})$, $7.51(d, J=16.0 \mathrm{~Hz}, 1 \mathrm{H},=\mathrm{CH}), 6.95(s, 2 \mathrm{H}$, arom. $)$, $6.35(d, J=16.0 \mathrm{~Hz}, 1 \mathrm{H},=\mathrm{CH}), 4.24(t, J=5.9 \mathrm{~Hz}$, $\left.2 \mathrm{H}, \mathrm{O}-\mathrm{CH}_{2}\right), 3.92(s, 6 \mathrm{H}, \mathrm{OMe}), 2.78(\mathrm{~d}, J=5.9 \mathrm{~Hz}$, $\left.2 \mathrm{H}, \mathrm{N}-\mathrm{CH}_{2}\right), 2.50\left(m, 4 \mathrm{H}, \mathrm{N}-\mathrm{CH}_{2}\right), 1.50\left(m, 2 \mathrm{H}, \mathrm{CH}_{2}\right)$ ppm;

${ }^{13} \mathrm{C}$ NMR (100 MHz, $\left.\mathrm{CDCl}_{3}\right): \delta=170.1\left(\mathrm{CO}_{2} \mathrm{R}\right)$, 147.5 (arom.), 145.1 (=CH), 137.1128 .3 (arom.), 115.9 (=CH), 105.9 (arom.), $62.7\left(\mathrm{O}-\mathrm{CH}_{2}\right), 56.1$ $(\mathrm{OMe}), 54.7\left(\mathrm{~N}-\mathrm{CH}_{2}\right), 54.2\left(\mathrm{~N}-\mathrm{CH}_{2}\right), 23.5\left(\mathrm{CH}_{2}\right), 22.9$ (CH2) ppm;

ESI-MS $(\mathrm{MeOH}): \mathrm{m} / \mathrm{z}(\%)=352-2\left([\mathrm{M}+\mathrm{H}]^{+}, 100\right)$, $374.2\left([\mathrm{M}+\mathrm{Na}]^{+}, 25\right)$;

analysis calcd for $\mathrm{C}_{18} \mathrm{H}_{25} \mathrm{NO}_{6}$ (335.40): C 64.46, H 7.51; found: C 64.03, H 7.81.

\section{Piperazine (7)}

This compound was commercially obtained from Merck and used as received.

\section{N-Boc-piperazine (8)}

This compound was prepared from 7 in $71 \%$ yield according to literature; m.p. $46-48^{\circ} \mathrm{C}$ (lit.: ${ }^{35} 46-$ $\left.47^{\circ} \mathrm{C}\right)$

\section{tert-Butyl 4-(2-hydroxyethyl)-piperazine-1-carbo- xylate (9)}

To an ice-cold solution of N-Boc-piperazine $(\mathbf{8}, 1.0 \mathrm{~g}$, $5.37 \mathrm{mmol})$ in dry DCM $(20 \mathrm{~mL})$, triethylamine $(1.09$ $\mathrm{g}, 10.77 \mathrm{mmol})$ and 2-bromo-ethanol $(95 \%, 1.34 \mathrm{~g}$, $10.19 \mathrm{mmol}$ ) were slowly added. The mixture was stirred at $60^{\circ} \mathrm{C}$ overnight. Usual aqueous work-up (water, brine, $\mathrm{MgSO}_{4}$ ) followed by column chromatography (silica gel, $\mathrm{CHCl}_{3} / \mathrm{MeOH}, 97: 3$ ) gave $9(865 \mathrm{mg}, 70 \%)$ as a colorless oil; $\mathrm{R}_{\mathrm{F}}=0.32$ (silica gel, $\left.\mathrm{CHCl}_{3} / \mathrm{MeOH}, 97: 3\right)$;

${ }^{1} \mathrm{H}$ NMR (400 MHz, $\left.\mathrm{CDCl}_{3}\right): \delta=3.53(t, J=5.2 \mathrm{~Hz}$, $\left.\mathrm{N}-\mathrm{CH}_{2}\right), 3.33\left(t, J=5.2 \mathrm{~Hz}, 4 \mathrm{H}, \mathrm{N}-\mathrm{CH}_{2}\right), 3.15(s, 1 \mathrm{H}$, $\mathrm{OH}), 2.44\left(t, J=5.2 \mathrm{~Hz}, 2 \mathrm{H}, \mathrm{N}-\mathrm{CH}_{2}\right), 2.35(t, J=5.2$ $\left.\mathrm{Hz}, 4 \mathrm{H}, \mathrm{N}-\mathrm{CH}_{2}\right), 1.34(s, 9 \mathrm{H}, \mathrm{Me}) \mathrm{ppm}$;

${ }^{13} \mathrm{C} \mathrm{NMR}\left(100 \mathrm{MHz}, \mathrm{CDCl}_{3}\right): \delta=154.5(\mathrm{C}=\mathrm{O}), 79.6$ $\left(\mathrm{C}_{\mathrm{q}}\right)$, 59.9, 57.8, $52.7\left(\mathrm{CH}_{2}\right), 28.3(\mathrm{Me}) \mathrm{ppm}$;

ESI-MS $(\mathrm{MeOH}): \mathrm{m} / \mathrm{z}(\%)=231.0\left([\mathrm{M}+\mathrm{H}]^{+}, 100\right)$, $131.1\left([\mathrm{M}+\mathrm{H}-\mathrm{Boc}]^{+}, 11\right)$;

analysis calcd for $\mathrm{C}_{11} \mathrm{H}_{22} \mathrm{~N}_{2} \mathrm{O}_{3}$ (230.31): C 57.31, H 9.63, N 12.16; found: C 57.11, H 9.97, N 11.97.

(E) tert-Butyl 4-[2-[(3-(3,5-dimethoxy-4-(triisopropylsilyl)oxy)phenyl]acryloyl)oxy)ethyl)piperazine-1-carboxylate (10) 
Following the procedure given for the preparation of 15, from 3 (1.0 g, $2.95 \mathrm{mmol})$ and 9 (0.908 g, 3.94 mmol) $10(1.28 \mathrm{~g}, 73 \%)$ was obtained as a colorless solid; m.p. $112-114^{\circ} \mathrm{C} ; \mathrm{R}_{\mathrm{F}}=0.66$ (silica gel, hexane/ethyl acetate/triethylamine, 100:50:3);

IR (KBr): $v=3448 b r, 2944 w, 2865 m, 1702 s, 1633 m$, $1581 m, 1511 s, 1461 m, 1424 m, 1285 m, 1246 m, 1159 s$, $1128 \mathrm{~s}, 1006 \mathrm{~m}, 906 \mathrm{~m} \mathrm{~cm}^{-1}$; UV/vis $(\mathrm{MeOH}): \lambda_{\max }(\log$ $\varepsilon)=249$ (4.29), $364(4.38) \mathrm{nm}$;

${ }^{1} \mathrm{H} \mathrm{NMR}\left(400 \mathrm{MHz}, \mathrm{CDCl}_{3}\right): \delta=7.59(d, J=15.8 \mathrm{~Hz}$, $=\mathrm{CH}), 6.71(s, 2 \mathrm{H}$, arom. $), 6.30(d, J=15.8 \mathrm{~Hz},=\mathrm{CH})$, $4.33\left(m, 2 \mathrm{H}, \mathrm{O}-\mathrm{CH}_{2}\right), 3.81(s, 6 \mathrm{H}, \mathrm{OMe}), 3.44(m, 4 \mathrm{H}$, $\left.\mathrm{N}-\mathrm{CH}_{2}\right), 2.71\left(m, 2 \mathrm{H}, \mathrm{N}-\mathrm{CH}_{2}\right), 2.48\left(m, 4 \mathrm{H}, \mathrm{N}-\mathrm{CH}_{2}\right)$, $1.46(s, 9 \mathrm{H}, \mathrm{Me}), 1.31-1.18$ (sept., $J=15.8 \mathrm{~Hz}, 3 \mathrm{H}$, $\mathrm{CH}), 1.08(d, J=15.8 \mathrm{~Hz}, 18 \mathrm{H}, \mathrm{Me}) \mathrm{ppm}$;

${ }^{13} \mathrm{C}$ NMR (100 MHz, $\left.\mathrm{CDCl}_{3}\right): \delta=167.0\left(\mathrm{CO}_{2} \mathrm{R}\right)$, 154.7 (NCO), 151.5 (arom.), $145.5(=\mathrm{CH}), 137.6$, 126.5 (arom.), 115.5 (=CH), 105.1 (arom.), $79.6\left(\mathrm{C}_{\mathrm{q}}\right)$, $61.6\left(\mathrm{O}-\mathrm{CH}_{2}\right), 56.8\left(\mathrm{~N}-\mathrm{CH}_{2}\right), 55.5(\mathrm{OMe}), 53.1(\mathrm{~N}-$ $\left.\mathrm{CH}_{2}\right), 28.4(\mathrm{Me}), 17.8(\mathrm{CH}), 13.3(\mathrm{Me}) \mathrm{ppm}$;

ESI-MS $(\mathrm{MeOH}): \mathrm{m} / \mathrm{z}(\%): 593.2\left([\mathrm{M}+\mathrm{H}]^{+}, 100\right)$, $615.2\left([\mathrm{M}+\mathrm{Na}]^{+}, 4\right)$;

analysis calcd for $\mathrm{C}_{31} \mathrm{H}_{52} \mathrm{~N}_{2} \mathrm{O}_{7} \mathrm{Si}$ (592.85): $\mathrm{C} 62.81, \mathrm{H}$ 8.84, N 4.73; found: C 62.66, H 9.03, N 4.47.

\section{(E) 3,4,5-Trimethoxy-cinnamic acid (11)}

This compound was commercially obtained from Merck and used as received.

\section{(E) 2-(Piperidin-1-yl)ethyl 3-(3,4,5-trimethoxy-} phenyl)-acrylate (12)

Following the procedure given for the preparation of 15, from $11(2.0 \mathrm{~g}, 8.39 \mathrm{mmol})$ and $4(1.83 \mathrm{~g}, 14.16$ mmol) $12(2.52 \mathrm{~g}, 86 \%)$ was obtained as a colorless solid; m.p. $49-51{ }^{\circ} \mathrm{C} ; \quad \mathrm{R}_{\mathrm{F}}=0.37$ (silica gel, hexane/ethyl acetate/triethylamine, 100:50:3);

IR (KBr): $v=3442 b r, 2938 m, 2784 w, 1708 s, 1581 m$, $1506 \mathrm{~m}, 1464 \mathrm{~m}, 1244 \mathrm{~s}, 1129 \mathrm{~s}, 1004 \mathrm{~m} \mathrm{~cm}^{-1}$; UV/vis $(\mathrm{MeOH}): \lambda_{\max }(\log \varepsilon)=242(4.31), 333(4.29) \mathrm{nm}$;

${ }^{1} \mathrm{H} \mathrm{NMR}\left(400 \mathrm{MHz}, \mathrm{CDCl}_{3}\right): \delta=7.59(d, J=15.9 \mathrm{~Hz}$, $=\mathrm{CH}), 6.74(s, 2 \mathrm{H}$, arom. $), 6.36(d, J=15.9 \mathrm{~Hz}, 1 \mathrm{H}$, $=\mathrm{CH}), 4.32\left(t, J=6.1 \mathrm{~Hz}, 2 \mathrm{H}, \mathrm{O}-\mathrm{CH}_{2}\right), 3.88(\mathrm{~s}, 6 \mathrm{H}$, $\mathrm{OMe}), 3.87(s, 3 \mathrm{H}, \mathrm{OMe}), 2.67(t, J=6.1 \mathrm{~Hz}, 2 \mathrm{H}, \mathrm{N}-$ $\left.\mathrm{CH}_{2}\right), 2.47\left(t, J=5.4 \mathrm{~Hz}, 4 \mathrm{H}, \mathrm{N}-\mathrm{CH}_{2}\right), 1.60(m, 4 \mathrm{H}$, $\left.\mathrm{CH}_{2}\right), 1.43\left(m, 2 \mathrm{H}, \mathrm{CH}_{2}\right) \mathrm{ppm}$;

${ }^{13} \mathrm{C}$ NMR $\left(100 \mathrm{MHz}, \mathrm{CDCl}_{3}\right): \delta=166.8(\mathrm{CO} 2 \mathrm{R})$, $153.4,144.7,140.1,129.8$ (arom.), $117.3(=\mathrm{CH})$, 105.2 (arom.), $62.0\left(\mathrm{~N}-C H_{2}\right), 60.9$ (OMe), $57.4(\mathrm{O}-$ $\left.\mathrm{CH}_{2}\right), 56.1(\mathrm{OMe}), 54.7\left(\mathrm{~N}-\mathrm{CH}_{2}\right), 25.8\left(\mathrm{CH}_{2}\right), 24.1$ $\left(\mathrm{CH}_{2}\right)$ ppm; ESI-MS (MeOH): $\mathrm{m} / \mathrm{z}(\%)=350.1$ $([\mathrm{M}+\mathrm{H}]+, 100)$;

analysis calcd for $\mathrm{C}_{19} \mathrm{H}_{27} \mathrm{NO}_{5}$ (349.43): $\mathrm{C} 65.31, \mathrm{H}$ 7.79, N 4.01; found: C 65.04, H 7.50, N 3.83.

(E) tert-Butyl 4-[2-[(3-(3,4,5-trimethoxyphenyl)acryloyl)oxy]ethyl]piperazine-1-carboxylate (13) Following the procedure given for the preparation of 15, from 11 (500 mg, $2.1 \mathrm{mmol}$ ) and $\mathbf{9}(725 \mathrm{mg}, 3.15$ mmol) 13 (577 mg, 61\%) was obtained as a colorless liquid; b.p. $203^{\circ} \mathrm{C}\left(1\right.$ bar); $\mathrm{R}_{\mathrm{F}}=0.32$ (silica gel, hexane/ethyl acetate/triethylamine, 100:50:3);
IR (film): $v=3446 b r, 2939 w, 1698 s, 1636 m, 1583 m$, $1505 m, 1420 m, 1276 s, 1246 s, 1171 s, 1128 s, 1004 m$ $\mathrm{cm}^{-1}$; UV/vis $(\mathrm{MeOH}): \lambda_{\max }(\log \varepsilon)=242$ (4.33), 340 (4.32) $\mathrm{nm}$;

${ }^{1} \mathrm{H}$ NMR $\left(400 \mathrm{MHz}, \mathrm{CDCl}_{3}\right): \delta=7.56(d, J=16.0 \mathrm{~Hz}$, $1 \mathrm{H},=\mathrm{CH}), 6.72(s, 2 \mathrm{H}$, arom. $), 6.33(d, J=16.0 \mathrm{~Hz}$, $1 \mathrm{H},=\mathrm{CH}), 4.30\left(m, 2 \mathrm{H}, \mathrm{O}-\mathrm{CH}_{2}\right), 3.85(s, 6 \mathrm{H}, \mathrm{OMe})$, $3.84(s, 3 \mathrm{H}, \mathrm{OMe}), 3.41\left(m, 4 \mathrm{H}, \mathrm{N}-\mathrm{CH}_{2}\right), 2.68(m, 2 \mathrm{H}$, $\left.\mathrm{N}-\mathrm{CH}_{2}\right), 2.45\left(m, 4 \mathrm{H}, \mathrm{N}-\mathrm{CH}_{2}\right), 1.42(s, 9 \mathrm{H}, \mathrm{Me}) \mathrm{ppm}$; ${ }^{13} \mathrm{C}$ NMR (100 MHz, $\left.\mathrm{CDCl}_{3}\right): \delta=166.7\left(\mathrm{CO}_{2} \mathrm{R}\right)$, 154.6 (NCO), 153.4 (arom.), $145.0(=\mathrm{CH}), 140.1$, 129.7 (arom.), 117.0 (=CH), 105.2 (arom.), $79.6\left(\mathrm{C}_{\mathrm{q}}\right)$, $61.7\left(\mathrm{O}-\mathrm{CH}_{2}\right), 60.9(\mathrm{OMe}), 56.8\left(\mathrm{~N}-\mathrm{CH}_{2}\right), \quad 56.1$ (OMe), $53.1\left(\mathrm{~N}-\mathrm{CH}_{2}\right), 28.4(\mathrm{Me}) \mathrm{ppm}$;

ESI-MS $(\mathrm{MeOH}): \mathrm{m} / \mathrm{z}(\%)=451.1\left([\mathrm{M}+\mathrm{H}]^{+}, 100\right)$, $473.1\left([\mathrm{M}+\mathrm{Na}]^{+}, 11\right)$;

analysis calcd for $\mathrm{C}_{23} \mathrm{H}_{34} \mathrm{~N}_{2} \mathrm{O}_{7}$ (450.53): $\mathrm{C}$ 61.32, H 7.61, N 6.22; found: C 61.06, H 7.87, N 5.94.

\section{(E) Cinnamic acid (14)}

This compound was commercially obtained from Merck and used as received.

\section{(E) 2-(Piperidin-1-yl)-ethyl cinnamate (15)}

To a solution of cinnamic acid $(\mathbf{1 4}, 2.0 \mathrm{~g}, 13.5 \mathrm{mmol})$ in dry DCM (40 mL) 4 (2.6 g, $20.12 \mathrm{mmol})$ and EDC $(3.14 \mathrm{~g}, 20.23 \mathrm{mmol})$ and cat. amounts of DMAP were added, and the mixture was stirred for 1 day at room temperature. Usual aqueous workup followed by column chromatography (silica gel, hexane/ethyl acetate/triethylamine, 100:50:3) gave $\mathbf{1 5}$ (3.16 g, $90 \%$ ) as a slightly yellowish liquid; $\mathrm{R}_{\mathrm{F}}=0.49$ (silica gel, hexane/ethyl acetate/triethylamine, 100:50:3); b.p. $308^{\circ} \mathrm{C}$ (1 bar);

IR (film): $v=3408 b r, 3061 w, 3028 w, 2934 s, 2883 m$, $2753 m, 1713 s, 1637 s, 1450 m, 1309 s, 1169 s, 1020 m$, $980 \mathrm{~m}, 767 \mathrm{~s}, 684 \mathrm{~m} \mathrm{~cm}-1$; UV/vis $(\mathrm{MeOH}): \lambda_{\max }(\mathrm{log}$ $\varepsilon)=299(4.16) \mathrm{nm}$;

${ }^{1} \mathrm{H}$ NMR $\left(400 \mathrm{MHz}, \mathrm{CDCl}_{3}\right): \delta=7.68(d, J=16.0 \mathrm{~Hz}$, $1 \mathrm{H},=\mathrm{CH}), 7.51(d d, J=6.7,2.9 \mathrm{~Hz}, 2 \mathrm{H}$, arom.), 7.417.34 ( $m, 3 \mathrm{H}$, arom.), $6.46(d, J=16.0 \mathrm{~Hz}, 1 \mathrm{H},=\mathrm{CH})$, $4.33\left(t, J=6.1 \mathrm{~Hz}, 2 \mathrm{H}, \mathrm{O}-\mathrm{CH}_{2}\right), 2.68(t, J=6.1 \mathrm{~Hz}$, $\left.2 \mathrm{H}, \mathrm{N}-\mathrm{CH}_{2}\right), 2.47\left(t, J=5.4 \mathrm{~Hz}, 4 \mathrm{H}, \mathrm{N}-\mathrm{CH}_{2}\right), 1.59$ $\left(m, 4 \mathrm{H}, \mathrm{CH}_{2}\right), 1.43\left(m, 2 \mathrm{H}, \mathrm{CH}_{2}\right) \mathrm{ppm}$;

${ }^{13} \mathrm{C}$ NMR $\left(100 \mathrm{MHz}, \mathrm{CDCl}_{3}\right): \delta=166.9\left(\mathrm{CO}_{2} \mathrm{R}\right)$, 144.7 (=CH), 134.1, 130.2, 128.8, 128.0 (arom.), $118.1 \quad(=\mathrm{CH}), 62.1 \quad\left(\mathrm{~N}-\mathrm{CH}_{2}\right), 57.4\left(\mathrm{OCH}_{2}\right), 54.8$ $\left(\mathrm{N}-\mathrm{CH}_{2}\right), 52.9\left(\mathrm{CH}_{2}\right), 24.2\left(\mathrm{CH}_{2}\right) \mathrm{ppm}$;

ESI-MS $(\mathrm{MeOH}): \mathrm{m} / \mathrm{z}(\%)=260.2\left([\mathrm{M}+\mathrm{H}]^{+}, 100\right)$; analysis calcd for $\mathrm{C}_{16} \mathrm{H}_{21} \mathrm{NO}_{2}$ (259.35): C 74.10, H 8.16, N 5.40; found: C 73.84, H 8.32, N 5.11.

(E) tert-Butyl 4-[2-(cinnamoyloxy)ethyl]piperazine-1-carboxylate (16)

Following the procedure given for the preparation of 15, from 14 (200 mg, $1.35 \mathrm{mmol}$ ) and 9 (466 mg, 2.02 $\mathrm{mmol}$ ), 16 (301 mg, 62\%) was obtained as a colorless oil; b.p. $153^{\circ} \mathrm{C}\left(1\right.$ bar); $\mathrm{R}_{\mathrm{F}}=0.25$ (hexane/ethyl acetate/triethylamine, 100:50:3); 
IR (film): $v=3448 b r, 2975 w, 1696 s, 1421 m, 1247 m$, $1171 s, 1004 w \mathrm{~cm}^{-1}$; UV/vis $(\mathrm{MeOH}): \lambda_{\max }(\log \varepsilon)=$ 298 (4.2) nm;

${ }^{1} \mathrm{H}$ NMR (400 MHz, $\left.\mathrm{CDCl}_{3}\right): \delta=7.68(d, J=16 \mathrm{~Hz}$, $1 \mathrm{H},=\mathrm{CH}), 7.52,7.39-7.36(\mathrm{~m}, 5 \mathrm{H}$, arom. $), 6.44(d, J$ $=16.0 \mathrm{~Hz}, 1 \mathrm{H},=\mathrm{CH}), 4.33\left(\mathrm{~m}, 2 \mathrm{H}, \mathrm{O}-\mathrm{CH}_{2}\right), 3.43(\mathrm{~m}$, $\left.4 \mathrm{H}, \mathrm{N}-\mathrm{CH}_{2}\right), 2.71\left(m, 2 \mathrm{H}, \mathrm{N}-\mathrm{CH}_{2}\right), 2.48(m, 4 \mathrm{H}$, $\left.\mathrm{N}-\mathrm{CH}_{2}\right), 1.45(s, 9 \mathrm{H}, \mathrm{Me}) \mathrm{ppm}$;

${ }^{13} \mathrm{C}$ NMR (100 MHz, $\left.\mathrm{CDCl}_{3}\right): \delta=166.8\left(\mathrm{CO}_{2} \mathrm{R}\right)$, 154.7 (NCO), $145.0(=\mathrm{CH}), 134.3,130.3,128.8$, 128.0 (arom.), $117.9(=\mathrm{CH}), 79.6\left(\mathrm{C}_{\mathrm{q}}\right), 61.8\left(\mathrm{OCH}_{2}\right)$, $56.7\left(\mathrm{~N}-\mathrm{CH}_{2}\right), 53.7\left(\mathrm{~N}-\mathrm{CH}_{2}\right), 28.4(\mathrm{Me}) \mathrm{ppm}$; ESI-MS $(\mathrm{MeOH}): \mathrm{m} / \mathrm{z}(\%)=361.1([\mathrm{M}+\mathrm{H}]+, 100)$, $383.1\left([\mathrm{M}+\mathrm{Na}]^{+}, 22\right)$;

analysis calcd for $\mathrm{C}_{20} \mathrm{H}_{28} \mathrm{~N}_{2} \mathrm{O}_{4}$ (360.45): C 66.64, H 7.83, N 7.77; found: C 66.41, H 7.96, N 7.45.

\section{References}

1- A. Gunia-Krzyżak, K. Słoczyńska, J. Popiół, P. Koczurkiewicz, H. Marona, E. Pękala, Cinnamic acid derivatives in cosmetics: current use and future prospects, Int. J. Cosmet. Sci. 2018, 40, 356-366.

2- E. Pontiki, A. Peperidou, I. Fotopoulos, D. Hadjipavlou-Litina, Cinnamate hybrids: A unique family of compounds with multiple biological activities, Curr. Pharm. Biotechnol. 2018, 19, 1019-1048.

3- K. Rajendran, A. Anwar, N.A. Khan, M.R. Shah, R. Siddiqui, Trans-Cinnamic Acid Conjugated Gold Nanoparticles as Potent Therapeutics against Brain-Eating Amoeba Naegleria fowleri, ACS Chem. Neurosci. 2019, 10, 2692-2696.

4- S.A. Tishina, V.S. Stroylov, I.A. Zanyatkin, A.K. Melnikova, V.I. Muronetz, Y.Y. Stroylova, Cinnamic acid derivatives as the potential modulators of prion aggregation, Mendeleev Commun. 2017, 27, 493-494.

5- S. Ullah, D. Kang, S. Lee, M. Ikram, C. Park, Y. Park, S. Yoon, P. Chun, H.R. Moon, Synthesis of cinnamic amide derivatives and their antimelanogenic effect in $\alpha$-MSH-stimulated B16F10 melanoma cells, Eur. J. Med. Chem. 2019, 161, 78-92.

6- S. Ullah, C. Park, M. Ikram, D. Kang, S. Lee, J. Yang, Y. Park, S. Yoon, P. Chun, H.R. Moon, Tyrosinase inhibition and anti-melanin generation effect of cinnamamide analogues, Bioorg. Chem. 2019, 87, 43-55.

7- R. Wang, W. Yang, Y. Fan, W. Dehaen, Y. Li, H. Li, W. Wang, Q. Zheng, Q. Huai, Design and synthesis of the novel oleanolic acid-cinnamic acid ester derivatives and glycyrrhetinic acidcinnamic acid ester derivatives with cytotoxic properties, Bioorg. Chem. 2019, 88, 102951. doi: 10.1016/j.bioorg.2019.102951.

8- K. Yang, Y. Li, Q. Tang, L. Zheng, D. He, Synthesis, mitochondrial localization of fluorescent derivatives of cinnamamide as anticancer agents, Eur. J. Med. Chem. 2019, 170, 45-54.

9- I. R.S. Baliza, S.L.R. Silva, L.D. Santos, J.H.A. Neto, R.B. Dias, C.B.S. Sales, C.A.G. Rocha, M.B.P. Soares, A.A. Batista, D.P. Bezerra, Ruthenium Complexes With Piplartine Cause Apoptosis Through MAPK Signaling by a p53Dependent Pathway in Human Colon Carcinoma Cells and Inhibit Tumor Development in a Xenograft Model, Front. Oncol. 2019, 9, doi: 10.3389/fonc. 2019.00582

10- A. A. Farooqi, R. Attar, I. Yaylim, M.Z. Qureshi, M. Todorovska, G.S. Karatoprak, S. Najafi, U.Y. Sabitaliyevich, T.G. Zhenisovna, D.P. de Sousa, X.K. Lin, Piperlongumine as anticancer agent: The story so far about killing many birds with one stone, Cell. Mol. Biol. 2018, 64, 102-107.

11-K. Piska, A. Gunia-Krzyzak, P. Koczurkiewicz, K. Wojcik-Pszczola, E. Pekala, Piperlongumine (piplartine) as a lead compound for anticancer agents - Synthesis and properties of analogues: A mini-review, Eur. J. Med. Chem. 2018, 156, 13-20.

12-Y. H. Seo, J.K. Kim, J.G. Jun, Synthesis and biological evaluation of piperlongumine derivatives as potent anti-inflammatory agents, Bioorg. Med. Chem. Lett. 2014, 24, 5727-5730.

13-S. Sommerwerk, R. Kluge, D. Ströhl, L. Heller, A.E. Kramell, S. Ogiolda, P. Liebing, R. Csuk, Synthesis, characterization and cytotoxicity of new piplartine dimers, Tetrahedron 2016, 72, 1447-1454.

14- H. Wang, H. Jiang, C. Corbet, S. de Mey, K. Law, T. Gevaert, O. Feron, M. De Ridder, Piperlongumine increases sensitivity of colorectal cancer cells to radiation: Involvement of ROS production via dual inhibition of glutathione and thioredoxin systems, Cancer Lett. 2019, 450, 42-52.

15-Z. S. Deng, C.F. Li, D. Luo, P. Teng, Z.Y. Guo, X. Tu, K. Zou, D.C. Gong, A new cinnamic acid derivative from plant-derived endophytic fungus Pyronema sp., Nat. Prod. Res. 2017, 31, 2413-2419.

16- K. Misra, A. Nag, A. Sonawane, N-(2-Bromo-4fluorophenyl)-3-(3,4-dihydroxyphenyl)acrylamide (CPAM), a small catecholic amide as an antioxidant, anti-diabetic and antibacterial compound, RSC Adv. 2016, 6, 104632-104641.

17- N. Nowacka, R. Nowak, M. Drozd, M. Olech, R. Los, A. Malm, Antibacterial, Antiradical Potential and Phenolic Compounds of Thirty-One Polish Mushrooms, Plos One 2015, 10, e0140355; doi: 10.1371/journal.pone.0140355.

18-E. Sieniawska, R. Sawicki, M. Swatko-Ossor, A. Napiorkowska, A. Przekora, G. Ginalska, E. Augustynowicz-Kopec, The Effect of Combining Natural Terpenes and Antituberculous Agents against Reference and Clinical Mycobacterium tuberculosis Strains, Molecules 2018, 23, E176; doi: 10.3390/molecules23010176. 
19- G. R. Silveira, K.A. Campelo, G.R.S. Lima, L.P. Carvalho, S.S. Samarao, O. Vieira-da-Motta, L. Mathias, C. R.R. Matos, I.J.C. Vieira, E.J.T. de Melo, E.J. Maria, In Vitro Anti-Toxoplasma gondii and Antimicrobial Activity of Amides Derived from Cinnamic Acid, Molecules 2018, 23, E774; doi: 10.3390/molecules23040774.

20-S. T. Xu, D.H. Li, L.L. Pei, H. Yao, C.Q. Wang, H. Cai, H.Q. Yao, X.M. Wu, J.Y. Xu, Design, synthesis and antimycobacterial activity evaluation of natural oridonin derivatives, Bioorg. Med. Chem. Lett. 2014, 24, 2811-2814.

21-S. Ghafary, Z. Najafi, M. MohammadiKhanaposhtani, H. Nadri, N. Edraki, N. Ayashi, B. Larijani, M. Amini, M. Mahdavi, Novel cinnamic acid-tryptamine hybrids as potent butyrylcholinesterase inhibitors: Synthesis, biological evaluation, and docking study, Arch. Pharm. 2018, 351, e1800115; doi: 10.1002/ardp.201800115.

22- J. Gomez, M.J. Simirgiotis, B. Lima, J.D. Paredes, C.M.V. Gabutti, C. Gamarra-Luques, J. Borquez, L. Luna, G.H. Wendel, A.O. Maria, G.E. Feresin, A. Tapia, Antioxidant, Gastroprotective, Cytotoxic Activities and UHPLC PDA-Q Orbitrap Mass Spectrometry Identification of Metabolites in Baccharis grisebachii Decoction, Molecules 2019, 24, E1085; doi: 10.3390/molecules24061085.

23-S. L. Guzman-Gutierrez, A. Nieto-Camacho, J.I. Castillo-Arellano, E. Huerta-Salazar, G. Hernandez-Pasteur, M. Silva-Miranda, O. Arguello-Najera, O. Sepulveda-Robles, C.I. Espitia, R. Reyes-Chilpa, Mexican Propolis: A Source of Antioxidants and Anti-Inflammatory Compounds, and Isolation of a Novel Chalcone and epsilon-Caprolactone Derivative, Molecules 2018, 23, E334. doi: 10.3390/molecules23020334.

24- J. H. Kim, K.L. Chan, L.W. Cheng, Cinnamic Acid Analogs as Intervention Catysts for Overcoming Antifungal Tolerance, Molecules 2017, 22, E1783; doi:10.3390/molecules22101783.

25- A. Peperidou, E. Pontiki, D. Hadjipavlou-Litina, E. Voulgari, K. Avgoustakis, Multifunctional Cinnamic Acid Derivatives, Molecules 2017, 22, E1247; doi: 10.3390/molecules22081247.

26- K. Takao, K. Toda, T. Saito, Y. Sugita, Synthesis of Amide and Ester Derivatives of Cinnamic Acid and Its Analogs: Evaluation of Their Free Radical Scavenging and Monoamine Oxidase and Cholinesterase Inhibitory Activities, Chem. Pharm. Bull. 2017, 65, 1020-1027.
27-H. Miyachi, Design, synthesis, and structureactivity relationship study of peroxisome proliferator-activated receptor (PPAR) $\delta$-selective ligands, Curr. Med. Chem. 2007, 14, 2335-2343.

28-L. M. Wang, B. Waltenberger, E.M. PferschyWenzig, M. Blunder, X. Liu, C. Malainer, T. Blazevic, S. Schwaiger, J.M. Rollinger, E.H. Heiss, D. Schuster, B. Kopp, R. Bauer, H. Stuppner, V.M. Dirsch, A.G. Atanasov, Natural product agonists of peroxisome proliferator-activated receptor-gamma (PPARgamma): a review, Biochem. Pharmacol. 2014, $92,73-89$.

29- J. Wiemann, J. Karasch, A. Loesche, L. Heller, W. Brandt, R. Csuk, Piperlongumine B and analogs are promising and selective inhibitors for acetylcholinesterase, Eur. J. Med. Chem. 2017, 139, 222-231.

30-Z. Li, F.C.F. Ip, N.Y. Ip, R. Tong, Highly transSelective Arylation of Achmatowicz Rearrangement Products by Reductive $\gamma$-Deoxygenation and Heck-Matsuda Reaction: Asymmetric Total Synthesis of (-)-Musellarins A-C and Their Analogues, Chem. - Eur. J. 2015, 21, 11152-11157.

31-F. H. Clarke, G.B. Silverman, C.M. Watnick, N. Sperber, 3-Azaphenothiazine and dialkylaminoalkyl derivatives, J. Org. Chem. 1961, 26, 1126-1132.

32- J. R. Horton, C.B. Woodcock, Q. Chen, X. Liu, X. Zhang, J. Shanks, G. Rai, B.T. Mott, D.J. Jansen, S.C. Kales, M.J. Henderson, M. Cyr, K. Pohida, X. Hu, P. Shah, X. Xu, A. Jadhav, D.J. Maloney, M.D. Hall, A. Simeonov, H. Fu, P.M. Vertino, X. Cheng, Structure-Based Engineering of Irreversible Inhibitors against Histone Lysine Demethylase KDM5A, J. Med. Chem. 2018, 61, 10588-10601.

33-M. R. Stentzel, D.A. Klumpp, Functionalized fluorenes via dicationic electrophiles, Tetrahedron Lett. 2019, 60, 1675-1677.

34- W. Tian, J. Li, Z. Su, F. Lan, Z. Li, D. Liang, C. Wang, D. Li, H. Hou, Novel Anthraquinone Compounds Induce Cancer Cell Death through Paraptosis, ACS Med. Chem. Lett. 2019, 10, 732-736.

35- I. A. Moussa, S.D. Banister, C. Beinat, N. Giboureau, A.J. Reynolds, M. Kassiou, Design, Synthesis, and Structure-Affinity Relationships of Regioisomeric N-Benzyl Alkyl Ether Piperazine Derivatives as sigma-1 Receptor Ligands, J. Med. Chem. 2010, 53, 6228-6239. 